\title{
Tyrosine receptor kinase $B$ silencing inhibits anoikis-resistance and improves anticancer efficiency of sorafenib in human renal cancer cells
}

\author{
PENG ZHANG $^{1 *}$, ZENGSHU XING $^{2 *}$, XUECHAO LI ${ }^{1}$, YARONG SONG $^{1}$, \\ JUN ZHAO $^{1}$, YAJUN XIAO ${ }^{1}$ and YIFEI XING ${ }^{1}$ \\ ${ }^{1}$ Department of Urology, Union Hospital, Tongji Medical College, Huazhong University of Science and Technology, \\ Wuhan, Hubei 430022; ${ }^{2}$ Department of Urology, Haikou People's Hospital/Haikou Hospital Affiliated \\ to Xiangya School of Medicine, Central South University, Haikou, Hainan 570208, P.R. China
}

Received October 29, 2015; Accepted December 8, 2015

DOI: 10.3892/ijo.2016.3356

\begin{abstract}
Renal cell carcinoma (RCC) is the most common solid neoplasm of adult kidney, and the major treatment for metastatic RCC (mRCC) is molecular targeted therapy. Sorafenib, as a multi-targeted tyrosine kinase inhibitor (TKI), has significantly improved clinical outcomes of mRCC patients. However, complete or long-term remissions are rarely achieved due to intolerance to dose-related adverse effects. It is therefore, necessary to explore novel target molecules for treatment or to enhance the therapeutic efficiency of present TKI for mRCC treatment. Anoikis is a specific type of apoptosis that plays a vital physiological role in regulating tissue homoeostasis. Anoikis-resistance is of critical importance for metastasis of various human cancers including mRCC. However, the precise mechanisms on anoikis-resistance in $\mathrm{mRCC}$ are still unclear. Tyrosine receptor kinase $\mathrm{B}$ (TrkB) belongs to the Trk family of neurotrophin receptors. Previous investigations have implied that activation or overexpression of TrkB promoted proliferation, survival, angiogenesis, anoikis-resistance and metastasis in human cancers. Yet, the correlation between TrkB and anoikis-resistance in $\mathrm{mRCC}$ has rarely been reported. The aim of the present study was to explore the impact of TrkB on anoikis-resistance and targeted therapy in mRCC. Our data revealed that anoikis-resistant $\mathrm{ACHN}$ cells presented
\end{abstract}

Correspondence to: Professor Yifei Xing, Department of Urology, Union Hospital, Tongji Medical College, Huazhong University of Science and Technology, 1277 Jiefang Avenue, Wuhan, Hubei 430022, P.R. China

E-mail: yifei_xing@163.com

${ }^{*}$ Contributed equally

Key words: renal cell carcinoma, anoikis, invasion, tyrosine receptor kinase $\mathrm{B}$, sorafenib, Akt, ERK with tolerance to detachment-induced apoptosis, excessive proliferation and aggressive invasion, accompanied by upregulation of TrkB expression in contrast to parental cells. Furthermore, TrkB silencing caused apoptosis, inhibited proliferation, retarded invasion as well as improved anticancer efficiency of sorafenib in anoikis-resistant ACHN cells through inactivation of PI3K/Akt and MEK/ERK pathways. Our data may offer a novel potential therapeutic strategy for $\mathrm{mRCC}$.

\section{Introduction}

Renal cell carcinoma ( $\mathrm{RCC}$ ) is the most common solid neoplasm of adult kidney, accounting for $3 \%$ of all adult cancers (1). Approximately 20-30\% of new RCC patients are diagnosed with metastatic RCC (mRCC), and as many as $40 \%$ of patients with localized RCC may develop mRCC after radical surgery (2). The prognosis of mRCC patients is poor and limited treatment options are available (3). Currently, the major treatment for $\mathrm{mRCC}$ is molecular targeted therapy, which includes tyrosine kinase inhibitors (TKIs) and mammalian target of rapamycin (mTOR) inhibitors. As a multi-targeted TKI, sorafenib was the first targeted agent approved for the treatment of $\mathrm{mRCC}$ and significantly prolonged median progression-free survival in a randomized phase III trial for advance RCC (4-6). However, complete or long-term remissions are rarely accomplished due to intolerance of dose-related side-effects (7). Identifying novel target molecules is hence necessary to improve the clinical outcome for mRCC treatment.

Anoikis is a specific type of apoptosis induced by loss of cell adhesion or inappropriate cell adhesion (8). Anoikis plays a critically important physiological role in regulating tissue homoeostasis (9). Failure of anoikis execution could result in adherent cells surviving under suspension conditions or at ectopic sites. Anoikis-resistance is consequently an essential prerequisite of progression and metastasis of various human cancer types $(10,11)$. Anoikis-resistance is closely related to $\operatorname{mRCC}(12,13)$, while the exact mechanisms of anoikisresistance in $\mathrm{mRCC}$ remain unclear. 
Tyrosine receptor kinase B (TrkB) was first identified as a highly expressed protein-tyrosine kinase in the brain and subsequently found as the signaling receptor for brain-derived neurotrophic factor (BDNF), which played a vital role in the development and repair of the nervous system (14). Activation of TrkB promoted tumor cell proliferation, survival, angiogenesis, epithelial-mesenchymal transition (EMT), anoikis-resistance and metastasis through regulating specific signaling pathways including phosphoinositide 3-kinases (PI3K)/Akt and MEK/ERK (15-17). Numerous studies have revealed that overexpression of TrkB is associated with anoikis-resistance and increased metastasis in various human cancers, such as neuroblastoma (17), hepatic carcinoma (18), lung adenocarcinoma (19), colorectal (20) and pancreatic cancer (21). Nevertheless, the correlation between TrkB and anoikis-resistance of $\mathrm{mRCC}$ is rarely reported.

The present study explored the effects of TrkB on anoikisresistance and targeted therapy in mRCC. Our data indicated that anoikis-resistant ACHN cells were characterized with tolerance to detachment-induced apoptosis, excessive proliferation and aggressive invasion, along with upregulation of TrkB expression in contrast to parental ACHN cells. It was also shown that TrkB silencing promoted detachment-induced apoptosis, reduced proliferation and repressed invasion in anoikis-resistant ACHN cells via inhibiting activities of Akt and ERK. Moreover, TrkB silencing improved anticancer efficiency of sorafenib in anoikis-resistant ACHN cells through inactivating PI3K/Akt and MEK/ERK pathways. Our findings may offer a novel potential therapeutic strategy for mRCC.

\section{Materials and methods}

Pharmaceuticals. Sorafenib (Bayer, Leverkusen, Germany) was dissolved in dimethyl sulfoxide (DMSO; Sigma-Aldrich, St. Louis, MO, USA) as a $10 \mathrm{mM}$ stock solution and stored at $-20^{\circ} \mathrm{C}$ or diluted in cell culture medium to give the appropriate final concentrations.

Cell culture and establishment of anoikis-resistant ACHN cell model. Human renal cancer ACHN cell line was obtained from Shanghai Cell Bank, Chinese Academy of Sciences (Shanghai, China) and cultured in minimum essential medium (MEM; Gibco, Grand Island, NY, USA) containing $10 \%$ fetal bovine serum (FBS; Zhejiang Tianhang Biotechnology Co., Ltd., Hangzhou, China) and $1 \%$ penicillin/streptomycin (Gibco) at $37^{\circ} \mathrm{C}$ in $5 \% \mathrm{CO}_{2}$ incubator. To obtain anoikis-resistant cells, ACHN cells were continuously cultured in ultra-low attachment 6-well plates (Corning Life Sciences, Acton, MA, USA) for 10 days (22), then transferred into normal culture plates and attachment-cultured for 3 days: the re-adherent cells were anoikis-resistant. The morphology of ACHN cells was observed with an inverted phase contrast microscope (Olympus, Tokyo, Japan).

Transfection of small interference RNA (siRNA). Three targetspecific si-TrkBs and a scramble control siRNA (si-Ctrl) were synthesized by Guangzhou RiboBio Co., Ltd. (Guangzhou, China). The sequences of the siRNAs were as follows (sense and antisense): si-TrkB-1 (5'-CCGUCACCUUGACUUGU CU-3' and 5'-AGACAAGUCAAGGUGACGG-3'); si-TrkB-2
(5'-CCACGAACAGAAGUAAUGA-3' and 5'-UCAUUACUU CUGUUCGUGG-3'); si-TrkB-3 (5'-GCGCUUCAGUGGUU CUAUA-3' and 5'-UAUAGAACCACUGAAGGC-3'); si-Ctrl (5'-UUCUCCGAACGUGUCACGU-3' and 5'-ACGUGACAC GUUCGGAGAA-3'). Cells were seeded in 6-well plates at $30 \%$ confluency. Next day, appropriate amount of si-TrkBs or si-Ctrl and Lipofectamine 2000 transfection reagent (Invitrogen, Carlsbad, CA, USA) were diluted in $250 \mu 1$ of opti-MEM reduced-serum medium (Gibco) respectively, and incubated for $5 \mathrm{~min}$ at room temperature. Diluted siRNAs were added into diluted Lipofectamine 2000 and incubated for $20 \mathrm{~min}$ at room temperature. A total of $500 \mu \mathrm{l}$ of the mixture was added each well. The medium was replaced with fresh culture medium after $6 \mathrm{~h}$.

The 3-(4,5-dimethyl-2-thiazolyl)-2,5-diphenyl-2H-tetrazo-

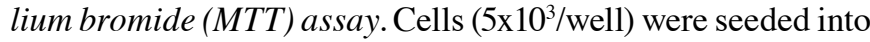
96-well plates and cultured in complete medium for $0,1,2,3$, 4, 5 and 6 days, respectively. A total of $20 \mu 1$ of MTT solution (Sigma-Aldrich) was added into each well and incubated for $4 \mathrm{~h}$ in the dark, and then $150 \mu \mathrm{l}$ of DMSO was added into each well for $10 \mathrm{~min}$. Absorbance was measured at $490 \mathrm{~nm}$ using a microtiter plate reader (Thermo Fisher Scientific, Waltham, MA, USA).

Colony formation assay. Isolated cells $\left(5 \times 10^{2} /\right.$ well) were seeded into 6-well plates and cultured in complete medium for 7 days. Cells were fixed with methanol and stained with $0.1 \%$ crystal violet (Sigma-Aldrich). Colonies with more than 50 cells were counted with an inverted phase contrast microscope.

Flow cytometry. Cell apoptosis was detected by Annexin V-FITC apoptosis detection kit (KeyGen Biotech., Co., Ltd., Nanjing, China). Cells (5x10\% $/$ well) were seeded in ultra-low attachment 6 -well plates for the indicated time, then collected for incubation with Annexin V-FITC and propidium iodide (PI) for $10 \mathrm{~min}$ in the dark at room temperature, and detected using a FACScan flow cytometer (BD Biosciences, San Jose, CA, USA).

Transwell assay. Cell invasion assay was performed using the 24-well Transwell plate with $8-\mu \mathrm{m}$ pore polycarbonate membrane inserts (Corning Life Sciences). Chamber inserts were coated with $50 \mu 1$ Matrigel (BD Biosciences, Bedford, MA, USA). Cells ( $3 \times 10^{4} /$ well) in $200 \mu 1$ serum-free medium were plated in the upper chambers, and $500 \mu$ l complete medium was added into the lower chambers. After incubation for $24 \mathrm{~h}$, cells that invaded into the lower surface of the membrane inserts were fixed in $4 \%$ paraformaldehyde, stained with $0.05 \%$ crystal violet and counted in 5 random fields with an inverted phase contrast microscope.

Quantitative real-time polymerase chain reaction ( $q R T-P C R$ ). Total RNA was extracted from cells using TRIzol reagent (Invitrogen) following the manufacturer's instruction. cDNA was synthesized from total RNA using First Strand cDNA Synthesis kit (Toyobo, Co., Ltd., Shanghai, China) following the manufacturer's protocol. qRT-PCR was performed with the SYBR-Green qPCR Mix (Toyobo) on the StepOnePlus Real-Time PCR system (Applied Biosystems, Foster City, CA, 
A

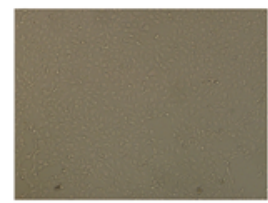

Parental

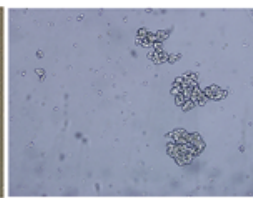

2

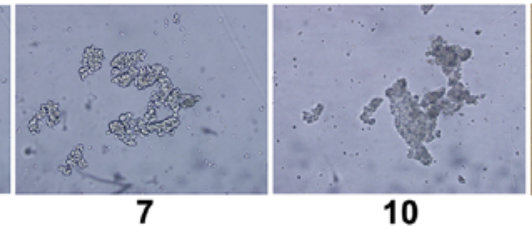

Suspension (days)

Re-adherent

B

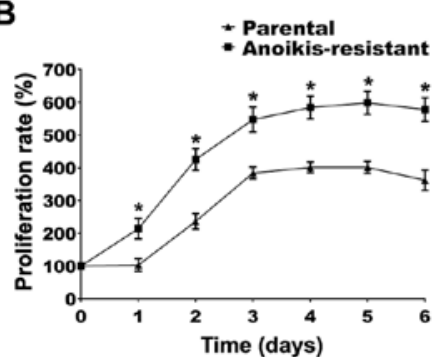

C
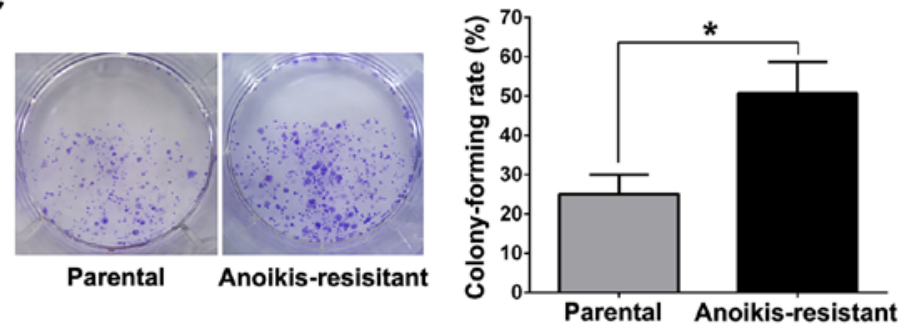

D

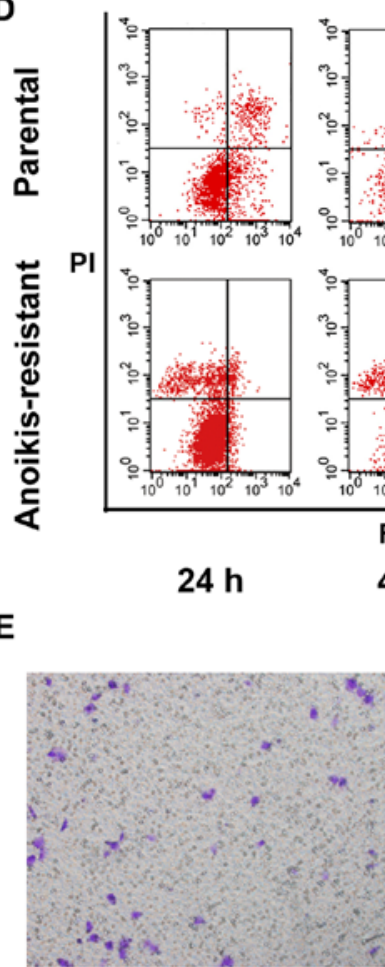

Parental
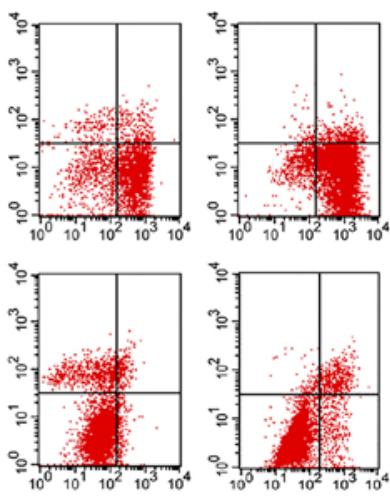

$72 \mathrm{~h}$

$48 \mathrm{~h}$
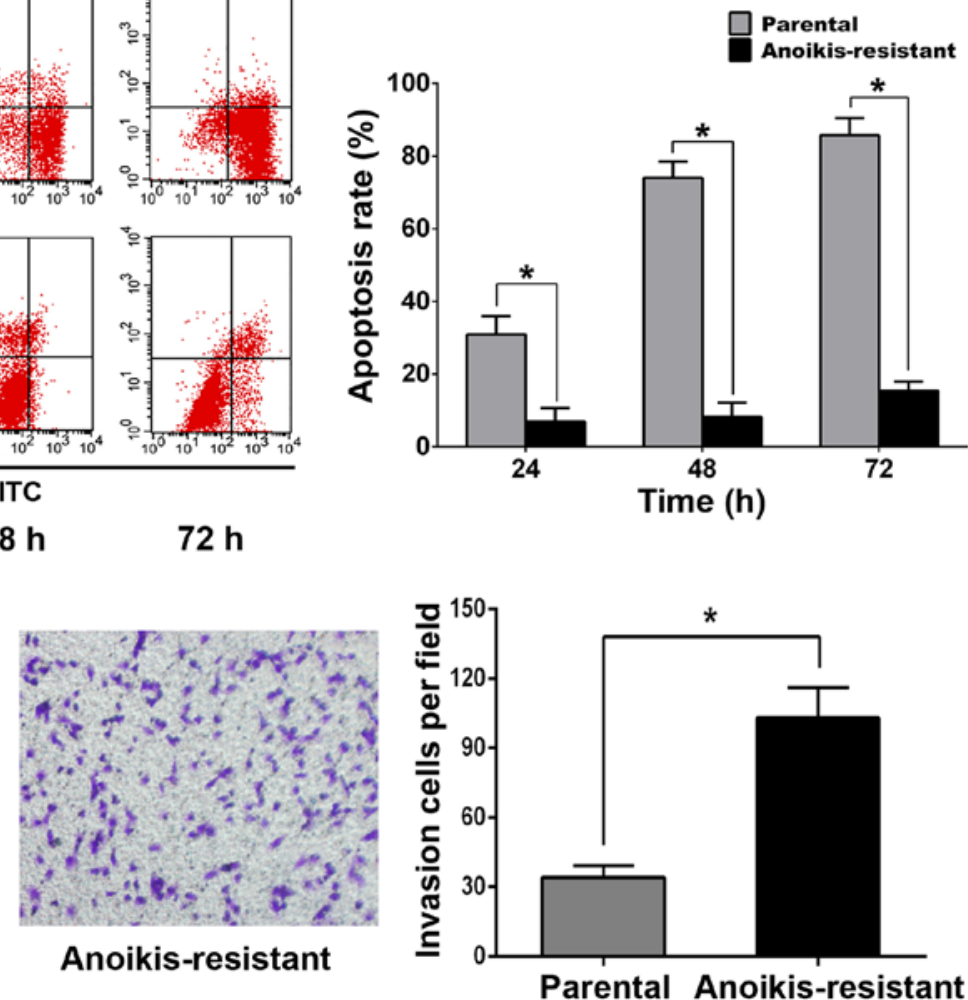

Figure 1. Anoikis-resistance inhibited detachment-induces apoptosis and promotes proliferation and invasion in ACHN cells. (A) The morphology of parental, suspension-cultured and re-adherent ACHN cells (original magnification, x200). (B) Parental or anoikis-resistant ACHN cells (5x10 $3 /$ well) were seeded in 96-well plates respectively; MTT was used to detect proliferation rates at the indicated time-points. (C) For colony formation assay, parental or anoikisresistant ACHN cells (5x10²/well) were seeded in 6-well plates for 7 days. (D) For flow cytometry, parental or anoikis-resistant ACHN cells (5x10 $/$ well) were cultured in ultra-low attachment 6-well plates, respectively, for the indicated time. (E) For Transwell assay, parental or anoikis-resistant ACHN cells $\left(3 \times 10^{4} /\right.$ well) in serum-free medium were seeded into upper chambers, respectively, and $500 \mu 1$ complete medium was added into lower chambers; invasion cells were counted after $24 \mathrm{~h}$ (original magnification, $\mathrm{x} 200$ ). ${ }^{*} \mathrm{P}<0.05$.

USA). The PCR primer pairs synthesized by Invitrogen and PCR programs were as follows: primer sequences (forward and reverse) for TrkB was 5'-GGGAACATCTCTCGGT CTATG-3' and 5'-CAAACTTGGAGTGTCTTGCC-3' and the following program was denaturation at $95^{\circ} \mathrm{C}$ for $60 \mathrm{sec}$, followed by 40 cycles consisting of denaturation at $95^{\circ} \mathrm{C}$ for $30 \mathrm{sec}$, annealing at $62^{\circ} \mathrm{C}$ for $20 \mathrm{sec}$, and extension at $72^{\circ} \mathrm{C}$ for $20 \mathrm{sec}$; primer sequences (forward and reverse) for internal control $\beta$-actin was 5'-GTCCACCGCAAATGCTTCTA-3' and 5'-TGCTGTCACCTTCACCGTTC-3' and the following program was denaturation at $95^{\circ} \mathrm{C}$ for $60 \mathrm{sec}$, followed by 40 cycles consisting of denaturation at $95^{\circ} \mathrm{C}$ for $30 \mathrm{sec}$, annealing at $56^{\circ} \mathrm{C}$ for $20 \mathrm{sec}$ and extension at $72^{\circ} \mathrm{C}$ for $20 \mathrm{sec}$. The results were analyzed using the $2^{-\Delta \Delta \mathrm{CT}}$ method.

Western blot analysis. Total proteins were isolated using radioimmunoprecipitation assay (RIPA) lysis buffer (Beyotime Institute of Biotechnology, Nanjing, China) and 

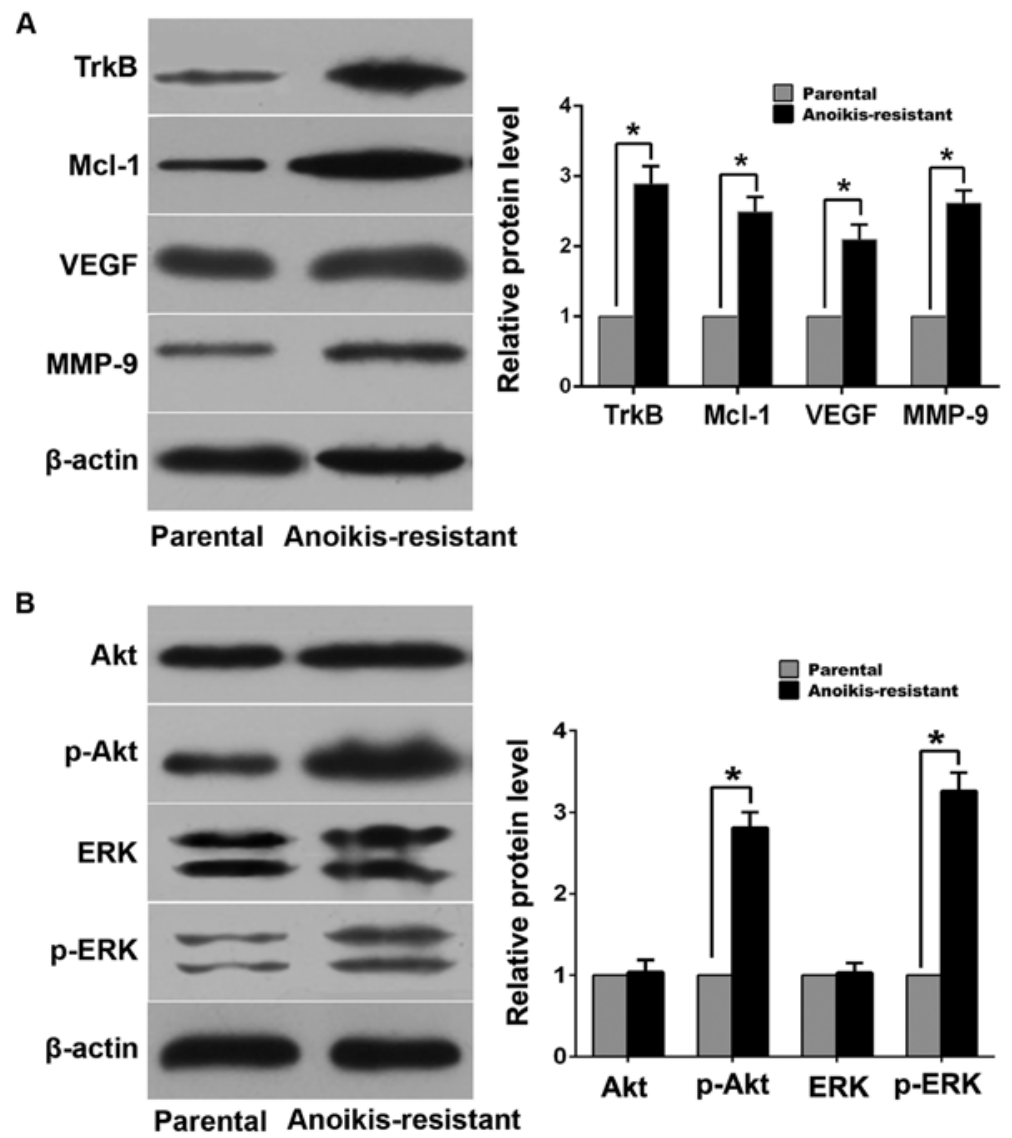

Figure 2. Expression of apoptosis- and metastasis-related genes and TrkB (A) was upregulated and activated Akt and ERK (B) were elevated in anoikisresistant ACHN cells. Protein expression was detected by western blot analysis and relative protein levels were analyzed by gray values of the bands. "P<0.05.

separated by sodium dodecyl sulfate (SDS)-polyacrylamide (PAGE) gels (Wuhan Boster Biological Technology, Ltd., Wuhan, China) and transferred onto nitrocellulose filter membranes (Millipore, Bedford, MA, USA). The membranes were blocked with 5\% non-fat milk in Tris-buffered saline with Tween-20 (TBST) and incubated with the primary antibodies overnight at $4{ }^{\circ} \mathrm{C}$. Primary antibodies were as follows: polyclonal rabbit anti-human TrkB (1:500), Mcl-1 (1:1,000), VEGF (1:500), Akt (1:1,000), p-Akt $(1: 1,000)$ and monoclonal rabbit anti-human $\beta$-actin $(1: 1,000)$ were purchased from Santa Cruz Biotechnology (Santa Cruz, CA, USA); polyclonal rabbit anti-human ERK $(1: 1,000)$ and p-ERK $(1: 1,000)$ were purchased from Bioworld Technology, Inc. (St. Louis Park, MN, USA); polyclonal rabbit anti-human MMP-9 (1:1,000) was purchased from Cell Signaling Technology (Beverly, MA, USA). $\beta$-actin was used as the loading control. The membranes were incubated with the horseradish peroxidase (HRP)-conjugated goat anti-rabbit secondary antibody (1:3,000; Santa Cruz Biotechnology). Bands were visualized with enhanced chemiluminescence (Beyotime Institute of Biotechnology). Densitometry was performed using the ImageJ software (National Institutes of Health, Bethesda, MA, USA).

Statistical analysis. All experiments were carried out at least in triplicate. Data were expressed as the mean \pm standard deviation (SD). Statistical analyses were performed using the Statistical Package for the Social Sciences (SPSS), version
17.0 (SPSS, Inc., Chicago, IL, USA). Statistical significances were evaluated using the Student's t-test or analysis of variance (ANOVA). ${ }^{*} \mathrm{P}<0.05$ was considered to indicate a statistically significant result.

\section{Results}

Anoikis-resistance inhibits detachment-induced apoptosis, promoting proliferation and invasion in ACHN cells. Anoikisresistant ACHN cell model was established according to previous reports (23-25). Briefly, parental ACHN cells were suspension-cultured in ultra-low attachment 6-well culture plates for 10 days. In suspension culture, parental ACHN cells gathered into clusters and gradually formed large cell masses with time (Fig. 1A). On the 10th day, ACHN cells were collected and attachment-cultured in normal culture plates, and the re-adherent cells were regarded anoikis-resistant. MTT, colony formation, flow cytometry and Transwell assays revealed that anoikis-resistant ACHN cells displayed more rapid proliferation, less detachment-induced apoptosis and greater capability of invasion compared with parental cells (Fig. 1B-E). Western blot analysis demonstrated that expression levels of myeloid cell leukemia-1 (Mcl-1), vascular endothelial growth factor (VEGF), matrix metalloproteinase-9 (MMP-9) and TrkB in anoikis-resistant ACHN cells were upregulated compared with parental cells (Fig. 2A). Additionally, expression of phosphorylated Akt (p-Akt) and phosphorylated ERK (p-ERK) increased in anoikis-resistant cells while expression 

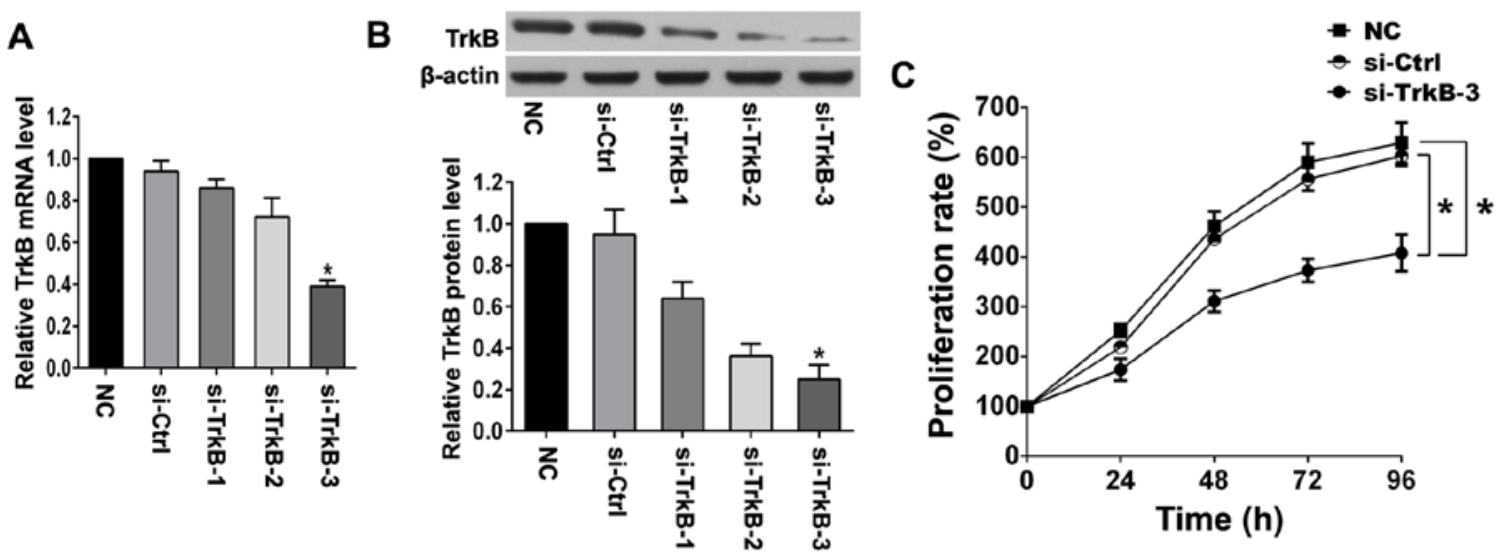

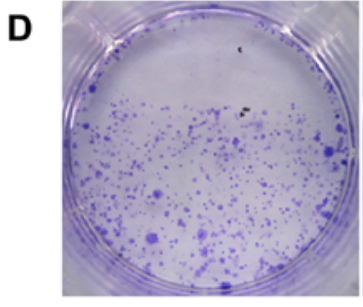

NC

E

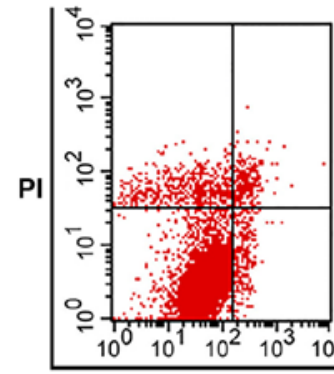

NC

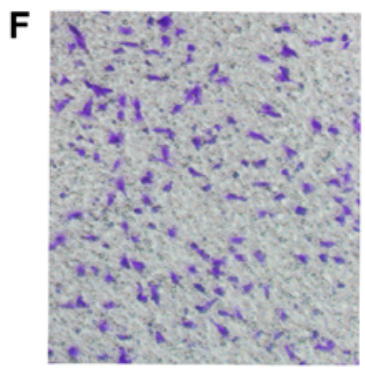

NC

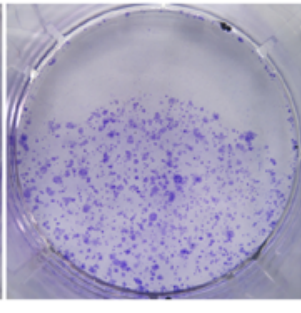

si-Ctrl

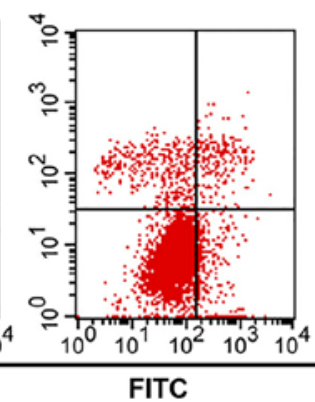

si-Ctrl

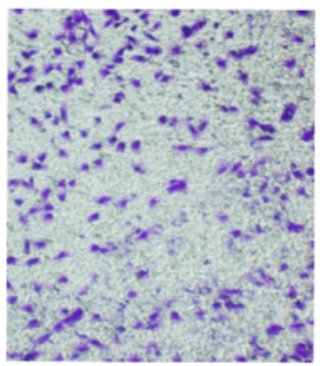

si-Ctrl

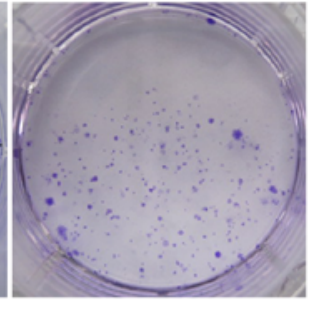

si-TrkB-3

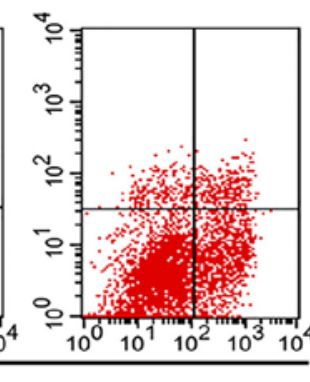

si-TrkB-3

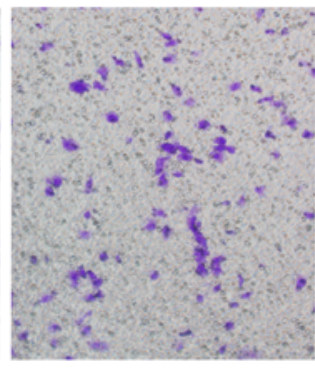

si-TrkB-3
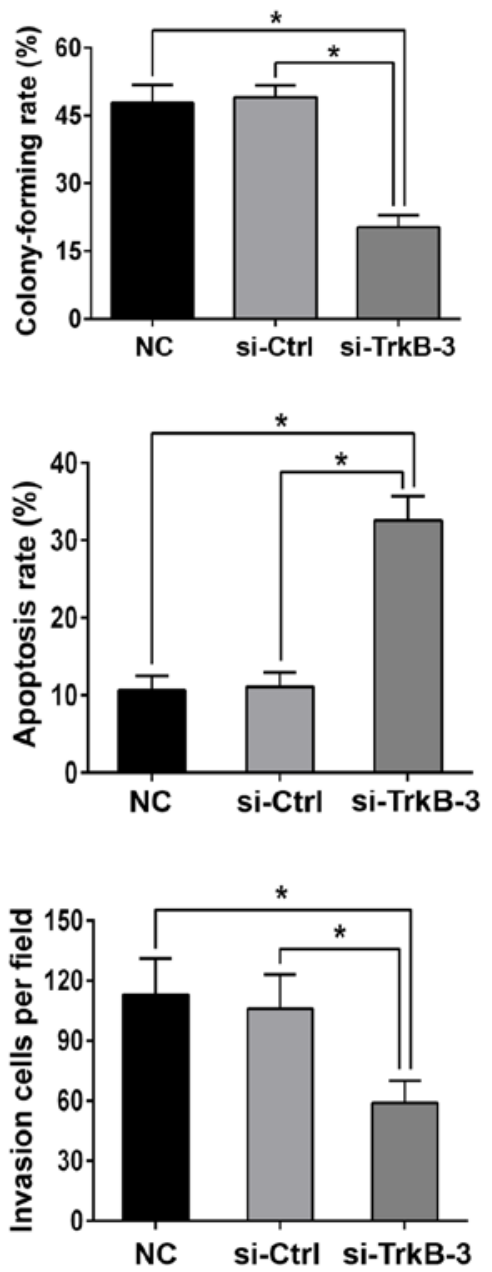

Figure 3. TrkB silencing promotes detachment-induced apoptosis as well as inhibits proliferation and invasion in anoikis-resistant ACHN cells. (A and B) TrkB silencing was verified with qRT-PCR and western blot analysis. (C) Anoikis-resistant ACHN cells (5x10 $3 /$ well) transfected without or with si-Ctrl and si-TrkB-3 for $48 \mathrm{~h}$ were seeded in 96-well plates, respectively; MTT was used to detect proliferation rates at the indicated time-points. (D) For colony formation assay, parental or anoikis-resistant ACHN cells (5x10²/well) transfected without or with si-Ctrl and si-TrkB-3 for 48 h were seeded in 6 -well plates for 7 days. (E) For flow cytometry, anoikis-resistant ACHN cells (5x105/well) transfected without or with si-Ctrl and si-TrkB-3 for $48 \mathrm{~h}$ were seeded in 96 -well plates respectively and apoptosis rates were detected after $72 \mathrm{~h}$. (F) For Transwell assay, anoikis-resistant ACHN cells (3x10 $4 /$ well) transfected without or with si-Ctrl and siTrkB-3 for $48 \mathrm{~h}$ were suspended in serum-free medium and seeded into upper chambers respectively, $500 \mu 1$ complete medium was added into lower chambers; invasion cells per field were counted after $24 \mathrm{~h}$. NC, negative control; si-Ctrl, scramble control siRNA; si-TrkB, TrkB siRNA (original magnification, x200). ${ }^{*} \mathrm{P}<0.05$.

of total Akt and ERK was of no significant difference between the two cell lines (Fig. 2B).

TrkB silencing promotes apoptosis, inhibits proliferation and reduces invasion in anoikis-resistant ACHN cells through inactivating Akt and ERK. Silencing efficiency of TrkB in anoikis-resistant ACHN cells was verified by qRT-PCR and western blot analysis (Fig. 3A and B). TrkB siRNA-3 (si-TrkB-3) showed the highest silencing efficiency and was chosen for the following experiments. Once TrkB was knocked 
A
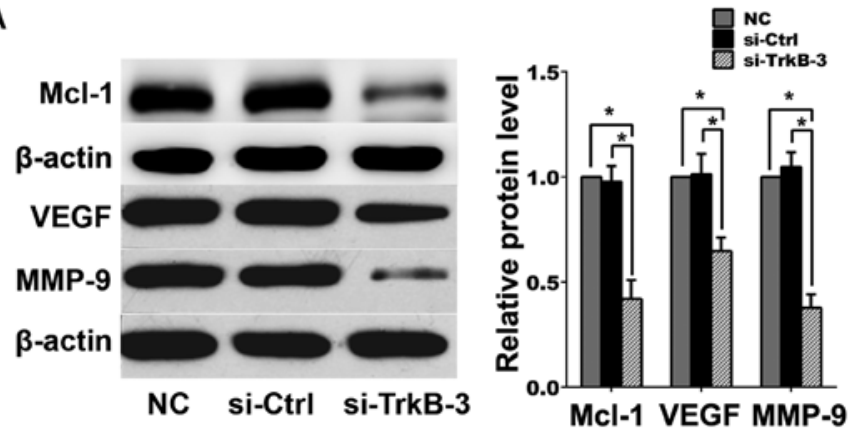

B
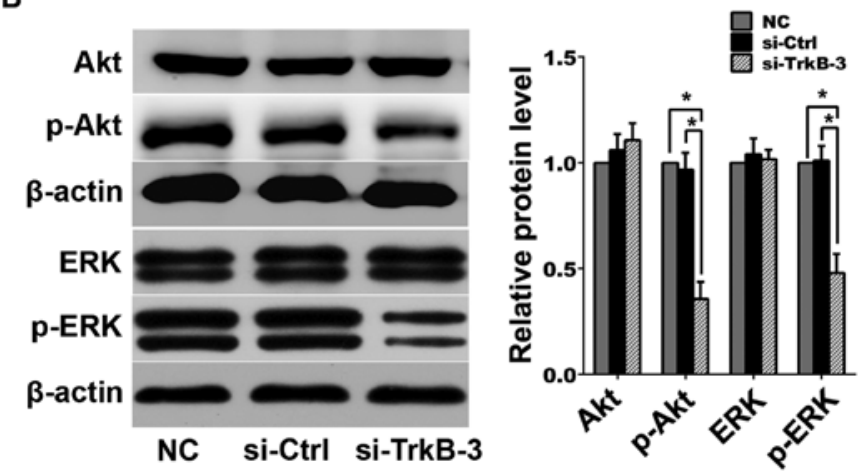

Figure 4. TrkB silencing downregulates expression of some apoptosis- and metastasis-related genes and inhibits the activity of Akt and ERK in anoikisresistant ACHN cells. (A and B) Protein expressions were detected by western blot analysis. Relative protein levels were analyzed by gray values of bands. ${ }^{*} \mathrm{P}<0.05$.

down, decreased proliferation rate, enhanced detachmentinduced apoptosis and diminished invasion were observed in anoikis-resistant ACHN cells (Fig. 3C-F). Western blot analysis indicated that following TrkB silencing, expression levels of Mcl-1, VEGF, MMP-9, p-Akt and p-ERK decreased in anoikis-resistant ACHN cells (Fig. 4).

TrkB silencing enhanced anticancer efficiency of sorafenib in anoikis-resistant ACHN cells through inhibiting activities of Akt and ERK. To investigate whether TrkB silencing enhanced anticancer efficiency of sorafenib in anoikis-resistant ACHN cells, $1.0 \mu \mathrm{M}$ was chosen as the sub-threshold concentration of sorafenib. There were no significant difference in proliferation, detachment-induced apoptosis and invasion between the groups without or with sorafenib (1.0 $\mu \mathrm{M})$ treatments (Fig. 5). However, combination of TrkB silencing and sorafenib $(1.0 \mu \mathrm{M})$ remarkably inhibited proliferation, reduced invasion and promoted detachment-induced apoptosis as compared with single treatment with TrkB knocked down (Fig. 5). In line with this, sorafenib $(1.0 \mu \mathrm{M})$ alone did not change the expressions of Mcl-1, VEGF, MMP-9 or the activity of Akt and ERK (Fig. 6), while combination of TrkB silencing and sorafenib $(1.0 \mu \mathrm{M})$ reduced the expression of Mcl-1, VEGF and MMP-9 significantly, as well as declined the expression of p-Akt and p-ERK, and such effects were more effective than treatment with TrkB silencing alone (Fig. 6).

\section{Discussion}

Establishment of anoikis-resistant cell model in vitro is usually on the basis of suspension culture that prevents cells from attachment (23-25). Therefore, we chose ultra-low attachment plates for the continuous suspension culture of the parental ACHN cells for 10 days, and the re-adherent cells were regarded as anoikis-resistant ACHN cells. Our data revealed that anoikis-resistant ACHN cells were characterized with more aggressive malignant biological behavior, including more rapid proliferation, less detachment-induced apoptosis and more capable of invasion in contrast to parental cells.

The molecular mechanisms involved in anoikis-resistance have been extensively investigated. PI3Ks are a family of lipid kinases that phosphorylate the 3'-OH group on phosphatidylinositols in the plasma membrane, resulting in recruitment of Akt to cell membrane for activation, which regulates tumor growth and survival (26). Likewise, MEK/ERK is the most typical mitogen activated protein kinase (MAPK) pathway, which controls cellular proliferation, invasion, differentiation and apoptosis, and aberrancy in the pathway contributes to malignant behavior (27). The activation or overexpression of PI3K/Akt and MEK/ERK pathways are supposed to promote anoikis-resistance in certain malignancies $(28,29)$. It was reported that activated Akt and ERK were associated with metastasis in RCC $(20,21)$. Our data confirmed that Akt and ERK activation are responsible for anoikis-resistance of ACHN cells and metastatic potential of RCC.

Previous studies have reported that anoikis is closely related to Bcl-2 family-mediated apoptosis pathway and Mcl-1 is an anti-apoptotic member (8). The combination of PI3K and MEK inhibition causes concomitant downregulation of Mcl-1 (26). Upregulation of Mcl-1 rendered anoikis-resistance in cancers, while downregulation of Mcl-1 increased sensitivity of cancer cells to anoikis (30). Similarly to those findings, 
A

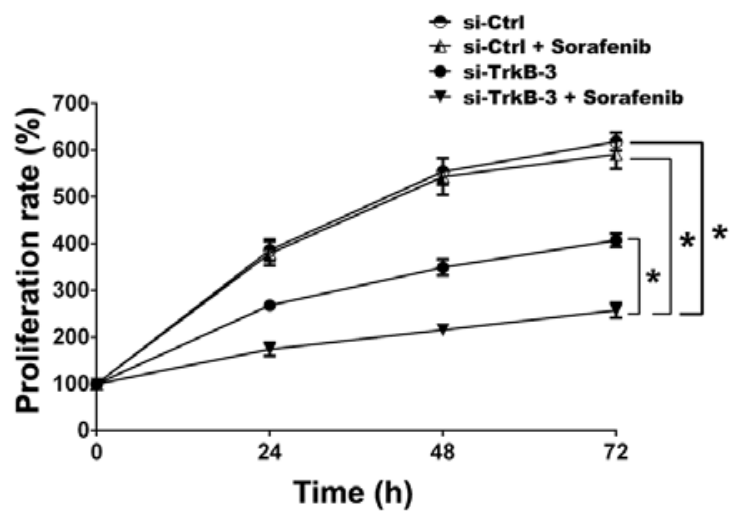

B

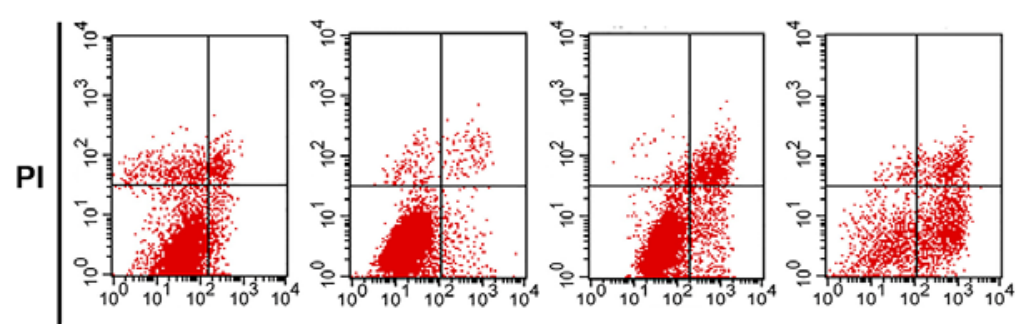

FITC
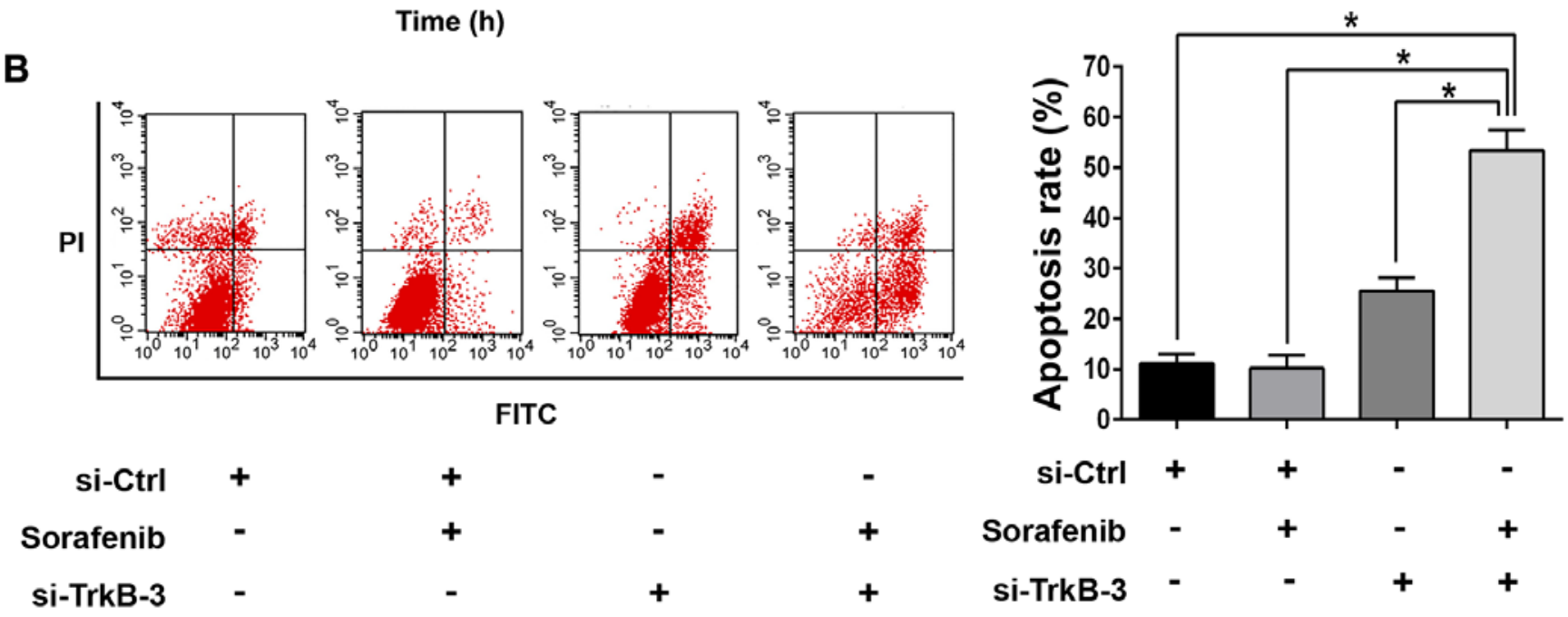

C
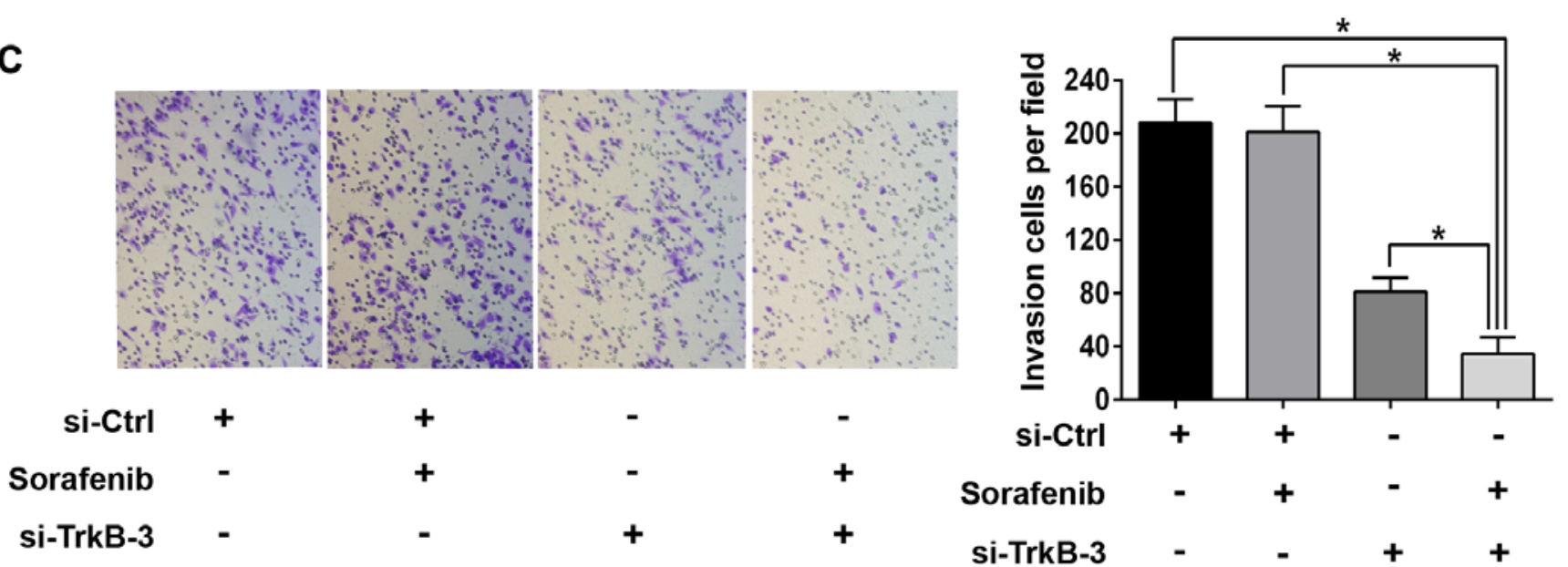

Figure 5. TrkB silencing enhances anticancer efficiency of sorafenib in anoikis-resistant ACHN cells. (A) Anoikis-resistant ACHN cells (5x10 $3 /$ well) transfected with si-Ctrl or si-TrkB-3 for $48 \mathrm{~h}$ were seeded in 96-well plates respectively, and sorafenib was added at the final concentration of $1.0 \mu \mathrm{M}$; MTT was used to detect proliferation rates at the indicated time-points. (B) For flow cytometry, anoikis-resistant ACHN cells (5x10 $/$ well) transfected with si-Ctrl or si-TrkB-3 were seeded in ultra-low attachment 6-well plates, and sorafenib was added at the final concentration of $1.0 \mu \mathrm{M}$; apoptosis rates were detected after 72 h. (C) For Transwell assay, anoikis-resistant ACHN cells (3x10 $/$ well) transfected with si-Ctrl or si-TrkB-3 for 48 h were suspended in serum-free medium and seeded into upper chambers, respectively, $500 \mu 1$ complete medium was added into lower chambers; sorafenib was added in upper and lower chambers at the final concentration of $1.0 \mu \mathrm{M}$; invasion cells per field were counted after $24 \mathrm{~h}$ (original magnification, $\mathrm{x} 200$ ). $\mathrm{P}<0.05$.

expression of Mcl-1 as well as metastasis-related genes VEGF and MMP-9 were upregulated in anoikis-resistant ACHN cells in the present study.

TrkB is closely correlated with tumor progression, anoikisresistance, metastasis and response to chemotherapy (17). Binding of BDNF to TrkB leads to auto-phosphorylation of tyrosines in the intracellular domain $(31,32)$. Overexpression of TrkB activates protein kinase B to block anoikis through PI3K signaling $(16,33)$. It was reported that Trk/PI3K/Akt pathway played an important role in anoikis-resistance and invasion of cancer cells (17). Activated TrkB induced EMT and enhanced tumor migration and invasion through MAPK-dependent Twist-Snail axis (34). The above evidence indicated that TrkB may serve as a potential therapeutic target for cancers. The present study illustrated that expression of TrkB was upregulated in anoikis-resistant ACHN cells, which implied 
A
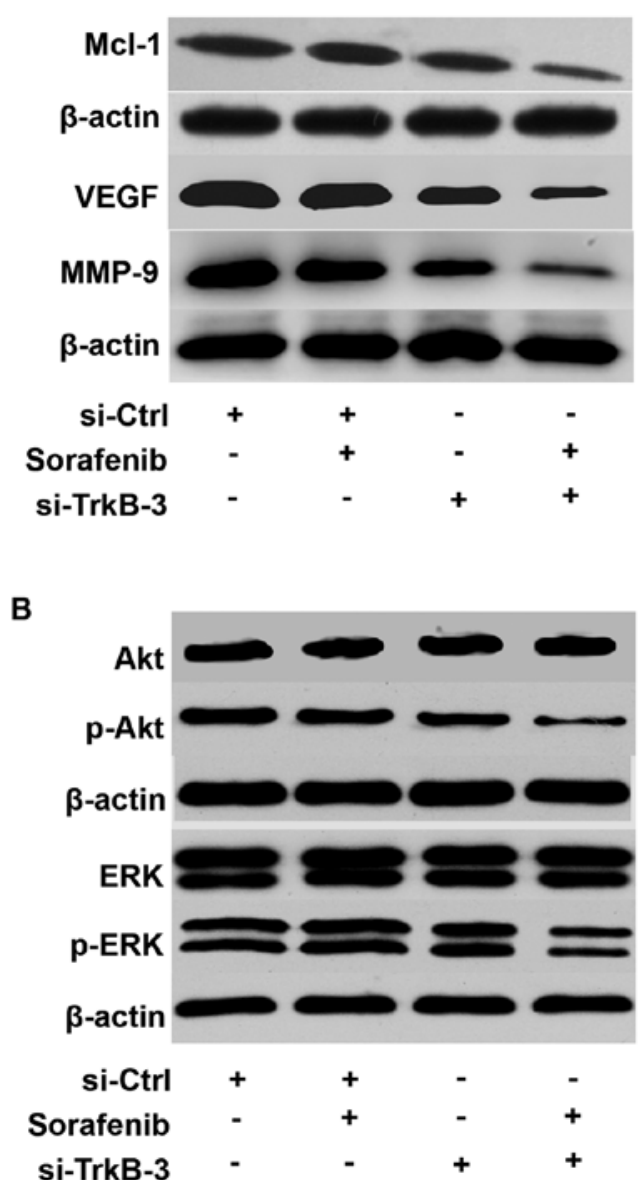
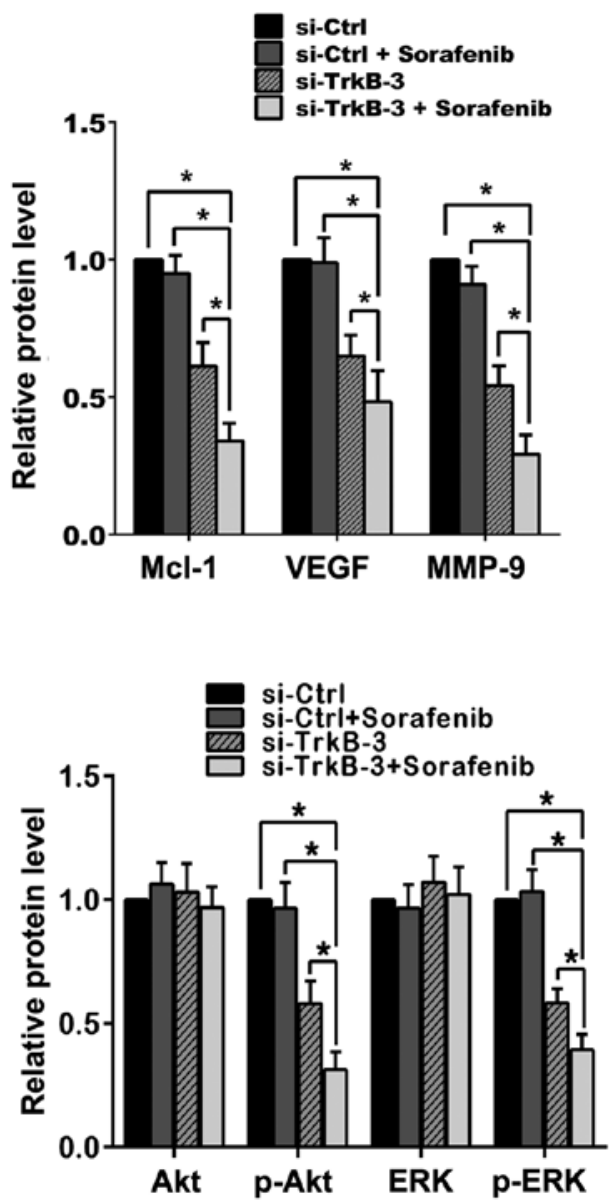

Figure 6. TrkB silencing enhances anticancer efficiency of sorafenib in anoikis-resistant ACHN cells through inhibiting the activity of Akt and ERK. (A and B) Protein expression was detected by western blot analysis and relative protein levels were analyzed by gray values of bands. ${ }^{*}<0.05$.

that TrkB might act as an oncogene. We revealed that TrkB silencing increased detachment-induce apoptosis, inhibited proliferation and invasion in anoikis-resistant ACHN cells, together with downregulated expression of Mcl-1, VEGF, MMP-9 and reduced activity of Akt and ERK.

Sorafenib is an orally administered small molecule TKI and has improved progression-free survival for advanced RCC patients (35). However, it presented only partial response rates partly due to dose-related adverse events and clinical toxicities (7,36,37). In this study, TrkB silencing enhanced anti-proliferative, pro-apoptotic and anti-invasive effects of sorafenib at a sub-threshold concentration in anoikis-resistant ACHN cells through inhibiting the activity of Akt and ERK. Based on our data, combination of TrkB silencing and sorafenib may be an alternative to clinical management of $\mathrm{mRCC}$ by enhancing the therapeutic effect of sorafenib and reducing dosage-dependent adverse events.

In summary, the present study revealed that TrkB was upregulated in anoikis-resistant ACHN cells, and silencing of TrkB reversed anoikis-resistance and inhibited invasion in ACHN cells; whereas, silencing of TrkB improved anticancer efficiency of sorafenib in anoikis-resistant ACHN cells through inactivating PI3K/Akt and MEK/ERK pathways. Our finding might offer a novel potential therapeutic strategy for $\mathrm{mRCC}$ and deserve further investigations.

\section{Acknowledgements}

The present study was supported by grants from the National Natural Science Foundation of China (nos. 30973008 and 81272847 to Y.X.) and the Program for New Century Excellent Talents in University of Ministry of Education of China (no. NCET-13-0239 to Y.X.).

\section{References}

1. Ljungberg B, Campbell SC, Choi HY, Jacqmin D, Lee JE, Weikert $S$ and Kiemeney LA: The epidemiology of renal cell carcinoma. Eur Urol 60: 615-621, 2011.

2. Kim DY, Karam JA and Wood CG: Role of metastasectomy for metastatic renal cell carcinoma in the era of targeted therapy. World J Urol 32: 631-642, 2014.

3. Ridge CA, Pua BB and Madoff DC: Epidemiology and staging of renal cell carcinoma. Semin Intervent Radiol 31: 3-8, 2014

4. Ratain MJ, Eisen T, Stadler WM, Flaherty KT, Kaye SB, Rosner GL, Gore M, Desai AA, Patnaik A, Xiong HQ, et al: Phase II placebo-controlled randomized discontinuation trial of sorafenib in patients with metastatic renal cell carcinoma. J Clin Oncol 24: 2505-2512, 2006.

5. Zustovich F, Lombardi G, Pastorelli D, Farina P, Bianco MD, De Zorzi L, Palma MD, Nicoletto O and Zagonel V: Clinical experience and critical evaluation of the role of sorafenib in renal cell carcinoma. Open Access J Urol 3: 69-82, 2011.

6. Escudier B, Eisen T, Stadler WM, Szczylik C, Oudard S, Siebels M, Negrier S, Chevreau C, Solska E, Desai AA, et al; TARGET Study Group: Sorafenib in advanced clear-cell renalcell carcinoma. N Engl J Med 356: 125-134, 2007. 
7. Bianchi L, Rossi L, Tomao F, Papa A, Zoratto F and Tomao S: Thyroid dysfunction and tyrosine kinase inhibitors in renal cell carcinoma. Endocr Relat Cancer 20: R233-R245, 2013.

8. Chiarugi P and Giannoni E: Anoikis: A necessary death program for anchorage-dependent cells. Biochem Pharmacol 76: 1352-1364, 2008.

9. Zhong X and Rescorla FJ: Cell surface adhesion molecules and adhesion-initiated signaling: Understanding of anoikis resistance mechanisms and therapeutic opportunities. Cell Signal 24: 393-401, 2012.

10. Simpson CD, Anyiwe K and Schimmer AD: Anoikis resistance and tumor metastasis. Cancer Lett 272: 177-185, 2008.

11. Jenning S, Pham T, Ireland SK, Ruoslahti E and Biliran H: Bit1 in anoikis resistance and tumor metastasis. Cancer Lett 333: 147-151, 2013.

12. Bouillez A, Gnemmi V, Gaudelot K, Hémon B, Ringot B, Pottier N, Glowacki F, Butruille C, Cauffiez C, Hamdane M et al: MUC1-C nuclear localization drives invasiveness of renal cancer cells through a sheddase/gamma secretase dependent pathway. Oncotarget 5: 754-763, 2014.

13. Sakamoto S, Schwarze S and Kyprianou N: Anoikis disruption of focal adhesion-Akt signaling impairs renal cell carcinoma. Eur Urol 59: 734-744, 2011

14. Stoilov P, Castren E and Stamm S: Analysis of the human TrkB gene genomic organization reveals novel TrkB isoforms, unusua gene length, and splicing mechanism. Biochem Biophys Res Commun 290: 1054-1065, 2002.

15. Glass DJ, Nye SH, Hantzopoulos P, Macchi MJ, Squinto SP Goldfarb M and Yancopoulos GD: TrkB mediates BDNF/NT-3dependent survival and proliferation in fibroblasts lacking the low affinity NGF receptor. Cell 66: 405-413, 1991.

16. Douma S, Van Laar T, Zevenhoven J, Meuwissen R, Van Garderen E and Peeper DS: Suppression of anoikis and induction of metastasis by the neurotrophic receptor TrkB. Nature 430: 1034-1039, 2004.

17. Thiele CJ, Li Z and McKee AE: On Trk - the TrkB signal transduction pathway is an increasingly important target in cancer biology. Clin Cancer Res 15: 5962-5967, 2009.

18. Lam CT, Yang ZF, Lau CK, Tam KH, Fan ST and Poon RT: Brain-derived neurotrophic factor promotes tumorigenesis via induction of neovascularization: implication in hepatocellular carcinoma. Clin Cancer Res 17: 3123-3133, 2011.

19. Sinkevicius KW, Kriegel C, Bellaria KJ, Lee J, Lau AN, Leeman KT, Zhou P, Beede AM, Fillmore CM, Caswell D, et al: Neurotrophin receptor TrkB promotes lung adenocarcinoma metastasis. Proc Natl Acad Sci USA 111: 10299-10304, 2014.

20. Fujikawa H, Tanaka K, Toiyama Y, Saigusa S, Inoue Y, Uchida K and Kusunoki M: High TrkB expression levels are associated with poor prognosis and EMT induction in colorectal cancer cells. J Gastroenterol 47: 775-784, 2012.

21. Sclabas GM, Fujioka S, Schmidt C, Li Z, Frederick WA, Yang W, Yokoi K, Evans DB, Abbruzzese JL, et al: Overexpression of tropomysin-related kinase B in metastatic human pancreatic cancer cells. Clin Cancer Res 11: 440-449, 2005.
22. Mawji IA, Simpson CD, Hurren R, Gronda M, Williams MA, Filmus J, Jonkman J, Da Costa RS, Wilson BC, Thomas MP, et al: Critical role for Fas-associated death domain-like interleukin-1-converting enzyme-like inhibitory protein in anoikis resistance and distant tumor formation. J Natl Cancer Inst 99: 811-822, 2007.

23. Fidler IJ: The pathogenesis of cancer metastasis: The 'seed and soil' hypothesis revisited. Nat Rev Cancer 3: 453-458, 2003.

24. Geiger TR and Peeper DS: The neurotrophic receptor TrkB in anoikis resistance and metastasis: A perspective. Cancer Res 65: 7033-7036, 2005

25. Liotta LA and Kohn E: Anoikis: Cancer and the homeless cell. Nature 430: 973-974, 2004.

26. Wong KK, Engelman JA and Cantley LC: Targeting the PI3K signaling pathway in cancer. Curr Opin Genet Dev 20: 87-90, 2010.

27. Montagut $\mathrm{C}$ and Settleman J: Targeting the RAF-MEK-ERK pathway in cancer therapy. Cancer Lett 283: 125-134, 2009.

28. Frisch SM and Screaton RA: Anoikis mechanisms. Curr Opin Cell Biol 13: 555-562, 2001.

29. Shih YW, Shieh JM, Wu PF, Lee YC, Chen YZ and Chiang TA: Alpha-tomatine inactivates PI3K/Akt and ERK signaling pathways in human lung adenocarcinoma A549 cells: effect on metastasis. Food Chem Toxicol 47: 1985-1995, 2009.

30. Wongpankam E, Chunhacha P, Pongrakhananon V, Sritularak B and Chanvorachote P: Artonin E mediates MCL1 down-regulation and sensitizes lung cancer cells to anoikis. Anticancer Res 32: 5343-5351, 2012

31. Klein R, Nanduri V, Jing SA, Lamballe F, Tapley P, Bryant S, Cordon-Cardo C, Jones KR, Reichardt LF and Barbacid M: The trkB tyrosine protein kinase is a receptor for brain-derived neurotrophic factor and neurotrophin-3. Cell 66: 395-403, 1991.

32. Pearse RN, Swendeman SL, Li Y, Rafii D and Hempstead BL: A neurotrophin axis in myeloma: TrkB and BDNF promote tumorcell survival. Blood 105: 4429-4436, 2005.

33. Yu X, Liu L, Cai B, He Y and Wan X: Suppression of anoikis by the neurotrophic receptor TrkB in human ovarian cancer. Cancer Sci 99: 543-552, 2008

34. Smit MA and Peeper DS: Zeb1 is required for TrkB-induced epithelial-mesenchymal transition, anoikis resistance and metastasis. Oncogene 30: 3735-3744, 2011.

35. Ramnath $\mathrm{N}$ and Adjei $\mathrm{A}$ : Inhibitors of Raf kinase and MEK signaling. Update Cancer Ther 2: 111-118, 2007.

36. Kim MJ, Kim DE, Jeong IG, Choi J, Jang S, Lee JH, Ro S, Hwang JJ and Kim CS: HDAC inhibitors synergize antiproliferative effect of sorafenib in renal cell carcinoma cells. Anticancer Res 32: 3161-3168, 2012.

37. Srikanthan A, Ethier JL, Ocana A, Seruga B, Krzyzanowska MK and Amir E: Cardiovascular toxicity of multi-tyrosine kinase inhibitors in advanced solid tumors: A population-based observational study. PLoS One 10: e0122735, 2015. 